\title{
Resequencing microarray for detection of human adenoviruses in patients with community-acquired gastroenteritis: a proof-of-concept study
}

Viral gastroenteritis is one of the most common infectious diseases worldwide, causing significant morbidity and mortality. The main groups of viruses associated with gastroenteritis in humans include rotaviruses, adenoviruses, noroviruses and astroviruses.

Traditionally, electron microscopic examination of stool specimens has been the main way to confirm the aetiology of viral gastroenteritis, as most of these viruses are difficult to culture. However, electron microscopic examination is labour-intensive and expensive. Although direct antigen detection is available for rotaviruses and adenoviruses, no comprehensive set of monoclonal antibodies against the other viruses is available. Nucleic acid detection can potentially overcome the above problems, and strategies such as multiplex PCR have been devised and validated to detect nucleic acids of multiple viruses simultaneously.

Another technology that has emerged in recent years to achieve the purpose of simultaneous detection of multiple viruses is the high-density resequencing microarray platform. In this technology, total nucleic acid extracted from samples is subjected to reverse transcription (RT) and multiplex PCR. The PCR products are pooled, purified, fragmented, end-labelled with biotin, hybridized to complementary sequences on microarray, and detected with fluorescent dye. It has been shown that such a high-density resequencing microarray is useful for detecting and tracking the evolution of viruses associated with respiratory infections (Wong et al., 2004; Lin et al., 2006, 2007; Wang et al., 2006). To our knowledge, no report on the usage of such high-density resequencing microarray platforms for the detection of viruses in stool specimens obtained from patients with gastroenteritis is available. In this proof-of-concept study, we examined the use of one of these platforms, TessArray Resequencing Pathogen Microarrays-Flu 3.1 (RPM-Flu-3.1; TessArae), originally designed for detection of viruses associated with respiratory infections, to detect human adenoviruses in stool specimens collected from patients with community-acquired gastroenteritis.

Forty-four prospectively collected, non-duplicated, stool specimens from paediatric patients under 16 years of age with community-acquired gastroenteritis sent to our laboratory during a 2-month (14 August-13 October 2008) period were included in the study. Communityacquired gastroenteritis is defined as the development of acute diarrhoea of three or more loose stools per day prior to or within $48 \mathrm{~h}$ after presentation to hospital. The male-to-female ratio was $13: 9$. The median age was 1 year (range 2 days-12 years). The stool specimens were subjected to bacterial culture, rotavirus antigen detection using the IDEIA Rotavirus kit (DAKO), human adenovirus PCR, norovirus RT-PCR and resequencing microarray analysis.

For resequencing microarray analysis, every four stool samples were pooled, resulting in 11 pooled samples, and subjected to total nucleic acid extraction and resequencing microarray analysis according to the manufacturer's instructions. DNA was extracted from all the 44 individual samples and human adenovirus PCR was performed followed by DNA sequencing in the case of positive samples (Okada et al., 2007). Phylogenetic tree construction was performed using the neighbour-joining method with CLUSTAL_X 1.83. For norovirus real-time RT-PCR, viral RNA was extracted from the stool specimens using the QIAamp Viral RNA Mini kit (Qiagen). RT was performed using the SuperScript II kit (Invitrogen). For real-time quantitative PCR assay,
cDNA was amplified with a LightCycler FastStart DNA Master HyProbes kit (Roche), using two pairs of forward specific primers (NLVG1-F, 5' -CGYTGGATGCGNTTYCATGA-3'; and NLVG2-F, 5'-CARGARBCNATGTTYAGRTGGATGAG-3') and reverse specific primers (NLVG1-R, 5' -CTTAGACGCCATCATCATTYAC-3'; and NLVG2-R, 5' -TCGACGCCATCTTCATTCACA-3' ${ }^{\prime}$ ) and $0.125 \mu \mathrm{M}$ of two specific probes [NLVG1-P, 5' (FAM)-AGATYGCGATCYCCTGTCCA(TAMAR)-3'; and NLVG2-P, 5' -(FAM)TGGGAGGGBGATCGCRATCT(TAMAR)-3'].

Bacterial culture was positive in eight patients. Rotavirus antigen was positive in one patient. Norovirus RT-PCR was positive in three patients.

Every four stool specimens were pooled and the 11 pooled samples were subjected to resequencing microarray analysis using the RPM-Flu-3.1 array. Among the 11 pooled samples, three were positive for human adenovirus, using both the TessArae server and NCBI BLAST service. No other virus was detected. For comparison, adenovirus PCR was positive in three stool specimens corresponding to the three positive pools. Sequencing results showed that the sequences fell into two distinct groups (Fig. 1). Two sequences (patients 36 and 44) were clustered with that of human adenovirus type 41, with a high bootstrap value of 1000; and the third sequence (patient 40) was clustered with that of human adenovirus type 21 , also with a high bootstrap value of 1000 (Fig. 1).

As there is no available resequencing microarray including all common viruses associated with acute conjunctivitis, we recently used the resequencing microarray for respiratory pathogens (RPM-Flu-3.1) to document that such a technology can be applied to virus detection in conjunctival swabs for patients with conjunctivitis 


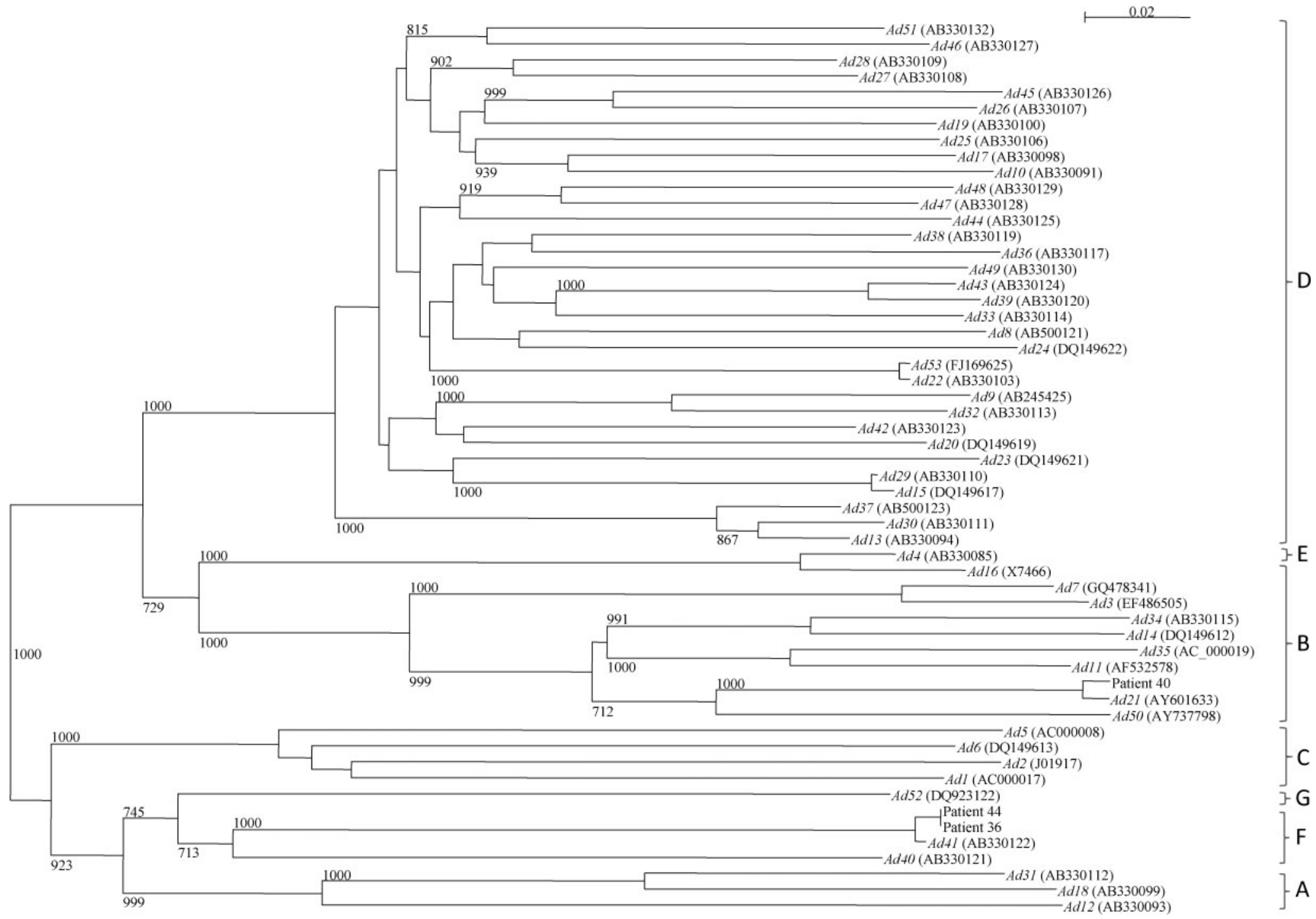

Fig. 1. Phylogenetic tree showing the relationships of the three human adenovirus sequences amplified from stool specimens from patients with community-acquired gastroenteritis to other human adenoviruses. The tree was constructed by the neighbour-joining method and rooted using the hexon gene sequence of bovine adenovirus (NC_006324). For analysis, 831 bases were used, and bootstrap values were calculated from 1000 trees. The scale bar indicates the estimated number of substitutions per 50 bases. Accession numbers are given as cited in the GenBank database. 
(Woo et al., 2010). In the present proof-ofconcept study, we used a similar approach to show that a resequencing microarray can also be applied to virus detection in stool specimens for patients with communityacquired gastroenteritis. The incidence of human adenovirus gastroenteritis in this cohort was about $7 \%$, which was similar to that in other studies on paediatric patients from different countries in Europe, Asia and Africa published in the last 5 years (median $3.3 \%$, range 1.9-19.9\%) (Li et al., 2005, 2009; Fabiana et al., 2007; Silva et al., 2008; Dey et al., 2009; Jin et al., 2009). Such a high sensitivity of the resequencing microarray, using PCR as the standard, for human adenoviruses has been shown for respiratory specimens, conjunctival swabs and stool specimens. This implies that a resequencing microarray is probably useful for most kinds of clinical specimens. Development of microarrays for community-acquired gastroenteritis, which should include not only adenoviruses, but also noroviruses, rotaviruses and astroviruses, can be performed for rapid diagnosis of the viral cause of gastroenteritis.

RPM-Flu-3.1, originally designed for respiratory viruses, is able to pick up different types of human adenoviruses. Although the panel of RPM-Flu-3.1 included only four human adenoviruses, type 2 (species C), 4 (species E), 7 (species B) and 17 (species D), it is able to pick up not only human adenoviruses types but also human adenovirus species that are not included in the panel. In our previous study, all the human adenoviruses were of species B and D (Woo et al., 2010). In the present study, the two strains of human adenovirus type 41 belonged to species $\mathrm{F}$, a human adenovirus species not included in the panel of RPM-Flu-3.1. It is notable that human adenovirus type 21 has only been found once in stool specimens from patients with gastroenteritis (Silva et al., 2008), but can also be picked up by RPMFlu-3.1. Unlike our previous study on patients with conjunctivitis, all three human adenoviruses in the present study were accurately assigned to the correct species of B (type 21) and F (type 41) by the TessArae server, although both human adenoviruses types 21 and 41 were not included in the panel of RPM-Flu-3.1.

The resequencing microarray allows detection of all viruses associated with a particular infectious disease syndrome simultaneously. This saves the labour of performing multiple PCRs/RT-PCRs for multiple viruses. In the present study, the cost of pooling the stool specimens would require Hong Kong dollars (HKD) 23595 for the 11 arrays and HKD 11616 for the consumables of the resequencing microarray analysis. Subsequent PCR of the 12 individual samples of the three positive pooled samples and DNA sequencing for the three individual positive samples were inexpensive (HKD 189). However, if all 44 individual samples were subjected to resequencing microarray analysis, it would require HKD 94380 and HKD 46464 for the 44 arrays and the associated consumables, respectively. Therefore, using the present strategy of pooling specimens for resequencing microarray analysis and subsequent PCR and DNA sequencing of individual specimens, $75 \%$ of the cost was saved as compared with resequencing microarray analysis of individual specimens, and this is not associated with any sacrifice of the sensitivity as demonstrated in both the present and our previous studies (Woo et al., 2010).

\section{Acknowledgements}

This work is partly supported by the Consultancy Service for Enhancing Laboratory Surveillance of Emerging Infectious Disease for Department of Health of the Hong Kong Special Administrative Region of China and Committee for Research and Conference Grant.

Patrick C. Y. Woo, ${ }^{1,2,3,4, \dagger}$

Susanna K. P. Lau, ${ }^{1,2,3,4, \dagger}$ Paul Lee, ${ }^{3}$ Ji Miao, ${ }^{3}$ Ami M. Y. Fung, ${ }^{3}$

Garnet K. Y. Choi, ${ }^{3}$

Rutledge Ellis-Behnke ${ }^{5,6}$ and KwokYung Yuen ${ }^{1,2,3,4}$

${ }^{1}$ State Key Laboratory of Emerging

Infectious Diseases, Hong Kong SAR

${ }^{2}$ Research Centre of

Infection and Immunology, The

University of Hong Kong, Hong Kong SAR

${ }^{3}$ Department of Microbiology, The University of Hong Kong, Hong Kong SAR

${ }^{4}$ Carol Yu Centre for Infection, The University of Hong Kong, Hong Kong SAR
${ }^{5}$ Department of Anatomy, The University of Hong Kong, Hong Kong SAR

${ }^{6}$ Department of Brain \& Cognitive Sciences, Massachusetts Institute of Technology, Cambridge, MA, USA

Correspondence: Kwok-Yung Yuen (hkumicro@hkucc.hku.hk)

†These authors contributed equally to this paper.

Dey, S. K., Shimizu, H., Phan, T. G., Hayakawa, Y., Islam, A., Salim, A. F., Khan, A. R., Mizuguchi, M., Okitsu, S. \& Ushijima, H. (2009). Molecular epidemiology of adenovirus infection among infants and children with acute gastroenteritis in Dhaka City, Bangladesh. Infect Genet Evol 9, 518-522.

Fabiana, A., Donia, D., Gabrieli, R., Petrinca, A. R., Cenko, F., Bebeci, D., Altan, A. M., Buonomo, E. \& Divizia, M. (2007). Influence of enteric viruses on gastroenteritis in Albania: epidemiological and molecular analysis. J Med Virol 79, 1844-1849.

Jin, Y., Cheng, W. X., Yang, X. M., Jin, M., Zhang, O., Xu, Z. O., Yu, J. M., Zhu, L., Yang, S. H. \& other authors (2009). Viral agents associated with acute gastroenteritis in children hospitalized with diarrhea in Lanzhou, China. J Clin Virol 44, 238-241.

Li, L., Phan, T. G., Nguyen, T. A., Kim, K. S., Seo, J. K., Shimizu, H., Suzuki, E., Okitsu, S. \& Ushijima, H. (2005). Molecular epidemiology of adenovirus infection among pediatric population with diarrhea in Asia. Microbiol Immunol 49, 121-128.

Li, C. S., Chan, P. K. \& Tang, J. W. (2009). Prevalence of diarrhea viruses in hospitalized children in Hong Kong in 2008. J Med Virol 81, 1903-1911.

Lin, B., Wang, Z., Vora, G. J., Thornton, J. A., Schnur, J. M., Thach, D. C., Blaney, K. M., Ligler, A. G., Malanoski, A. P. \& other authors (2006). Broad-spectrum respiratory tract pathogen identification using resequencing DNA microarrays. Genome Res 16, 527-535.

Lin, B., Blaney, K. M., Malanoski, A. P., Ligler, A. G., Schnur, J. M., Metzgar, D., Russell, K. L. \& Stenger, D. A. (2007). Using a resequencing microarray as a multiple respiratory pathogen detection assay. J Clin Microbiol 45, 443-452.

Okada, M., Ogawa, T., Kubonoya, H., Yoshizumi, H. \& Shinozaki, K. (2007). Detection and sequence-based typing of human adenoviruses using sensitive universal primer sets for the hexon gene. Arch Virol 152, 1-9.

Silva, P. A., Stark, K., Mockenhaupt, F. P., Reither, K., Weitzel, T., Ignatius, R., Saad, E., Seidu-Korkor, A., Bienzle, U. \& Schreier, E. (2008). Molecular characterization of enteric viral agents from children in northern region of Ghana. J Med Virol 80, 1790-1798. 
Wang, Z., Daum, L. T., Vora, G. J., Metzgar, D., Walter, E. A., Canas, L. C., Malanoski, A. P., Lin, B. \& Stenger, D. A. (2006). Identifying influenza viruses with resequencing microarrays. Emerg Infect Dis 12, 638-646.
Wong, C. W., Albert, T. J., Vega, V. B., Norton, J. E., Cutler, D. J., Richmond, T. A., Stanton, L. W., Liu, E. T. \& Miller, L. D. (2004). Tracking the evolution of the SARS coronavirus using high-throughput, high-density resequencing arrays. Genome Res 14, 398-405.
Woo, P. C., Lau, S. K., Choi, G. K., Fung, H. T., Shek, K. C., Miao, J., Chan, B. Y., Ng, K. H., Ngan, A. H. \& other authors (2010).

Resequencing microarray for detection of human adenovirus in patients with conjunctivitis. J Clin Virol 47, 282-285. 